\title{
The Effects of Gender Affirming Treatment on the Sporting Performance and Muscle Memory of Transgender Athletes. A Protocol for The Tavistock Transgender Athlete Study (TTAS).
}

\author{
Blair R. Hamilton*1-4, Giscard Lima ${ }^{1,5}$, James Barrett $^{3}$, Leighton Seal ${ }^{3}$, Alexander Kolliari-Turner ${ }^{1}$, \\ Fergus M. Guppy ${ }^{2,4}$ and Yannis P. Pitsiladis ${ }^{1,5}$ \\ ${ }^{1}$ Collaborating Centre of Sports Medicine, University of Brighton, Eastbourne, UK \\ ${ }^{2}$ Centre for Stress and Age-related Disease, University of Brighton, Brighton, UK \\ ${ }^{3}$ The Gender Identity Clinic Tavistock and Portman NHS Foundation Trust, London, UK \\ ${ }^{4}$ School of Pharmacy and Biomolecular Sciences, University of Brighton, Huxley Building, Lewes Road, Brighton, UK \\ ${ }^{5}$ Department of Movement, Human and Health Sciences, University of Rome "Foro Italico," Rome, Italy
}

Keywords: Transgender, Sport, Performance, Muscle Memory, Transwomen, Transmen.

\begin{abstract}
The issue of integrating transgender athletes into sport is becoming more prominent with the rising numbers of those identifying as transgender in society. Whether it is fair for transgender athletes to be included in their affirmed gender category across all levels of sport from grassroots to elite is the crux of the debate. Previous studies have shown muscle mass loss in transwomen and muscle mass and strength gain in transmen after 1 year of gender-affirming treatment (GAT). Wiik et al., 2020 found that transmen retain a strength disadvantage over cisgender men and transwomen retain muscle mass and strength advantages over cisgender women after 1 year of GAT. Roberts et al., 2020 also found that running performance in transwomen was maintained but not baseline muscular strength. However, very little data on sports performance measures outside muscular strength and running times exist, nor has any of the previous data been compared with a comparative control group. The aim of the TTAS is to investigate the effect of "muscle memory" in transgender athletes and investigate changes in physiology after 2 years of GAT such as bone mineral density, lean muscle mass and fat mass, coupled with sports performance measures in transwomen and transmen athletes and compare them with a cisgender female athletic cohort. This will elucidate what advantages/disadvantages transgender athletes gain/retain after 2 years of GAT over their cisgender counterparts and this will better inform policymakers who control their integration into their affirmed gender category in sport.
\end{abstract}

\footnotetext{
*Corresponding Author: Blair R. Hamilton (B.R.Hamilton@brighton.ac.uk) All authors have read and approved this version of the manuscript for pre-print. Authors BRH@blair90x or FMG @fergusguppy can be contacted on twitter.
}

Please cite as: Hamilton BR, Lima, G, Barrett, J, Seal, L, Kolliari-Turner, A, Guppy, FM and Pitsiladis YP. The Effects of Gender Affirming Treatment on the Sporting Performance and Muscle Memory of Transgender Athletes. A Protocol for The Tavistock Transgender Athlete Study (TTAS). SportRx iv.

DOI: 10.31236/osf.io/4rc2b 


\section{Introduction}

The clinical diagnosis of gender dysphoria in transgender people is when such individuals experience conflict between the gender that they were given during childbirth and their gender identity/experienced gender[1-3]. Some[3-5] but not all [2, 3, 6] will choose to undergo genderaffirming treatment (GAT) with cross-sex hormone replacement therapy alone or coupled with surgery to conform to their chosen gender. The number of transgender individuals who have been attending transgender health services has increased considerably in recent years in many European countries[7-9] and the issue of integrating transgender athletes is becoming more prominent with the rising numbers of those identifying as transgender in society.

On average, men will perform better than women, with the approximate sporting performance difference between men and women being $10-12 \%$ in favour of men on the evidence of World Athletics (then the IAAF) [10]. The issue with integrating transgender athletes into competitive sports is the debate over whether it is fair for transgender athletes to be included in their affirmed gender category across all levels of sport from grassroots to elite. The polarizing debate over fairness is especially highlighted in transwomen competing in the female category of sports, as it is vigorously debated that they possess unfair advantages due to exposure of high levels of testosterone during puberty[1, 11-13]. Very little progress has been made on this subject. The arguments used in 1976 that transwomen retain the muscular advantages of a male and genetically remained a male[14]are still being used 44 years later[15]. There has also been speculation and fear among sports organizations, female athletes, and spectators that men might try to pass as women in sports competitions to achieve victory[16], even though this has never come to pass.

It is worth noting the presence of high circulating testosterone concentrations does not guarantee increased performance, but the way an individual's body physiologically responds to testosterone does[14]. The argument against transwomen competing in female categories assumes that transwomen have benefitted from higher effective testosterone concentrations earlier in and throughout their life until the administration of GAT. However, the argument of hormonal advantages being interpreted as unfair in transwomen is specifically based on the fact that cisgender female 
competitors cannot naturally possess that same advantage[12] i.e. high testosterone concentrations during puberty or high testosterone during training periods before GAT. This argument is not present in transmen, as if a transman undergoes GAT through doctor prescribed testosterone replacement therapy, the endocrine society recommends that clinicians should maintain serum testosterone concentrations during treatment in the mid-normal range for healthy young men[17], therefore hypothetically not giving transmen potential competitive advantages of testosterone over cisgender men.

Previous studies have addressed the effects of androgen deprivation therapy and GAT in transwomen [18-22] and transmen[20, 22-24]. Loss of muscle mass has been shown in transwomen [19-22] and gains in muscle mass have been shown in transmen [20,22]. Early studies showed that gonadal suppression in men resulted in decreased muscular strength[18], however, a more recent study showed that transwomen had no change in muscle strength [22]. Transmen's muscular strength was also shown to improve throughout their treatment [22, 23]. The study by Wiik et al., 2020 [22] was the only one to compare their results with cisgender men and cisgender women and it was found that transmen retain a strength disadvantage over cisgender men and transwomen retain muscle mass and strength advantages over cisgender women after 1 year of GAT. However, this cohort of cisgender men $(n=17)$ and cisgender women $(n=14)$ was from a previous study investigating high doses of antiinflammatory drugs[25] and therefore, potentially not a comparative control group. This coupled with the relatively low number of transwomen $(n=11)$ and transmen $(n=12)$ highlights the need for further research to confirm these observations.

Recently, Roberts et al., 2020 [26] retrospectively reviewed pre- and post-GAT military fitness test results in transwomen $(\mathrm{n}=46)$ of the United States Air Force. The authors found that the push-up (31\% more than their female counterparts) and sit-up (15\% more than their female counterparts) advantages over ciswomen at baseline had been negated after 2 years of GAT, but not after 1 year. This finding agrees with previous studies that have shown that baseline muscular strength in transwomen is not significantly diminished after 1 year [22, 26] but after 2 years of GAT [26]. Roberts et al., 2020 also found that running performance in the 1.5 -mile run remained $12 \%$ faster on 
average in transwomen after 2 years of GAT [26]. These findings require replication in trained transwomen athletes, although they would suggest a different rate and extent of mitigation of the advantages held by transwomen given that strength advantages, but not cardiovascular advantages of transwomen were mitigated after 2 years of GAT. These observations also question the required testosterone suppression time of 1 year for transwomen to be eligible to compete in women's sport, as most advantages over ciswomen were not negated after 1 year of GAT. How applicable these performance data [26] are in determining the extent of advantage remaining in transwomen athletes' post-GAT remains to be determined, as this retrospective study failed to report hormone concentrations of the study participants and the training regimes of the participants. .

Another drawback of previous studies is the lack of data on sports performance measures outside muscular strength. Sporting performance measures such as $\dot{V}_{o_{2 m a x}}$ and strength should be studied within a cohort of transwomen and transmen and compared with a cisgender female control group to ascertain if there are any lasting advantages present. Bone health should also be studied, as only one study [24] has investigated this in transwomen and showed that they had reduced bone mineral density at the lumbar spine 3 years after the start of GAT. The extent of such bone mineral density loss needs to be determined as the lower the athletes bone mineral density, the greater the fracture risk to the athlete[27].

A central tenet of the "muscle memory" hypothesis is that the myonuclei that accumulate as a result of hypertrophy are retained during conditions of detraining/atrophy and for prolonged periods [28, 29]. However, due to conflicting data on myonuclear permanence, current evidence provides no consensus on the existence of muscle memory by myonuclear permanence and more research, particularly in humans, is warranted to experimentally test the muscle memory mechanism[30]. Transwomen, who have been exposed to higher circulating levels of testosterone during puberty than cisgender women, may have higher myonuclei per fibre values that are then retained for prolonged periods after the commencement of GAT (despite decrements in muscle mass) giving them a retained advantage that may be permanent [31]. Myonuclear permanence may also not be the predominant mechanism in the 
muscle memory phenomenon and that other mechanisms such as an epigenetic memory or residual miRNA levels may play a significant role[32, 33].

\section{Aim}

The aim of the Tavistock Transgender Athlete Study (TTAS) is two-fold:

Firstly, to investigate the effect of GAT on the myonuclei per fibre values in transgender athletes. Currently, no studies have investigated how myonuclei per fibre values change in transgender athletes, particularly those transwomen athletes who have experienced a high testosterone level earlier in their life and then subsequently undergo GAT. They will further enhance our understanding of the molecular basis of muscle memory and potentially allow us to harness these effects in clinical conditions involving muscle wastage and weakness - such as healthy ageing.

Secondly, the TTAS aims to investigate the changes in physiology and sports performance measures in transwomen and transmen athletes and compare them with a cisgender female athletic cohort. This will enhance our knowledge and elucidate the extent to which transgender athletes are at an advantage or disadvantage compared with comparative cisgender female athletic controls.

\section{Materials and Methods}

\subsection{Collaborating Centre}

The Tavistock Centre: The TTAS study is a unique collaboration founded in 2018 and established with the Tavistock Centre in Hammersmith, London, England (https://tavistockandportman.nhs.uk). The Tavistock has pioneered medical treatment for young transgender individuals and runs a specialist gender identity clinic that helps adults who experience difficulties with gender dysphoria. It is the largest gender clinic in Great Britain, being the size of all the others put together, and the only one that employs a consultant endocrinologist as part of the team.

\subsection{Study Participants}

The TTAS will investigate the changes that occur to sporting performance including muscle fibre physiology during 20 transwomen's and 20 transmen's transition and compare them to a cisgender 
female university athlete control arm, to establish what extent certain advantageous or disadvantageous muscle fibre parameters such as myonuclear number, satellite cell number, and fibre cross-sectional area (CSA) and typing from the vastus lateralis, as well as sporting performance measures such as strength and $\dot{\mathrm{V}}_{\mathrm{O}_{2} \mathrm{Max}}$ are altered during these transitions. The eligibility criteria for the transgender athlete arm is shown in Table 1 and the cisgender female control arm in Table 2. This will further elucidate if transitioning individuals retain an advantage after the 2 years of their GAT cycle (i.e., transwomen ) or gain significant advantages after 2 years of their GAT cycle (i.e., with the administration of testosterone in transmen). Study participants will be referred to the lead author by the collaborating centre and after informed verbal consent is given at the time of recruitment, written informed consent to take part in this research will be given on the first day of data collection before any data is collected from each participant.

Table 1. Eligibility criteria for the transgender athlete arm of the TTAS

* Healthy transmen or transwomen.

* Clinical diagnosis of Gender dysphoria.

* Have not yet undergone GAT.

* Aged 18-35 years old.

* Have a BMI in the range of $>18.5$ and $<30$.

* Physically active and/or play a competitive sport.

* No allergy to lidocaine

Table 2. Eligibility criteria for the cisgender female arm of the TTAS

$*$ Healthy Cisgender Female.
$*$ Aged $18-35$ years old.
$*$ Have a BMI in the range of $>18.5$ and $<30$
$*$ No allergy to lidocaine




\subsection{Study Design}

This longitudinal study will involve 5 participant visits for the transgender athlete arm shown in

Figure 1, to the School of Pharmacy and Biomolecular Sciences, University of Brighton, Huxley

Building, Lewes Road, Brighton, UK over 2 years. The cisgender female athlete control arm will only visit the centre once to provide control values.
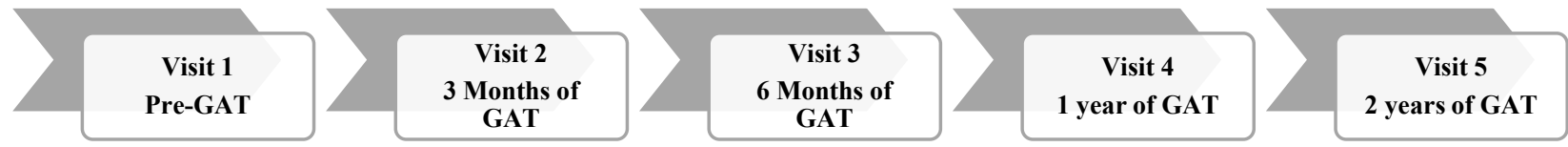

Figure 1. Longitudinal study design for the transgender athlete arm of the TTAS

\subsection{Ethical Approval}

Ethical approval for this protocol has been obtained from the Brighton and Sussex Research Ethics

Committee, with an amendment being sought to include the measure of Dual X-ray Absorptiometry

(DXA) scanning.

\subsection{Outcome measures}

Urine collection: $60 \mathrm{~mL}$ of urine will be collected into Sterilin ${ }^{\mathrm{TM}}$ Urine Bottles (Thermo Fisher Scientific, Loughborough, Leicestershire, UK). $1 \mathrm{~mL}$ aliquots will be then taken from this bottle and stored in 3 x $1.5 \mathrm{~mL}$ TubeOne ${ }^{\circledR}$ Microcentrifuge Tubes (Star Lab, Milton Keynes, Buckinghamshire, UK). Both these aliquots and the urine bottle will be stored at $-80^{\circ} \mathrm{C}$ until subsequent analysis.

Blood collection: Blood will be collected by a trained phlebotomist from an antecubital vein utilizing a closed vacuette system. A $3 \mathrm{~mL}$ whole blood sample will be collected into a Tempus ${ }^{\mathrm{TM}}$ Blood RNA Tube (Life Technologies, Carlsbad, CA, USA) to preserve RNA for subsequent extraction, a $10 \mathrm{~mL}$ whole blood sample will be collected into a $\mathrm{BD}{ }^{\circledR}$ serum tube (Becton, Dickinson, and Company, Wokingham, Berkshire, UK) for serum extraction and a $10 \mathrm{~mL}$ whole blood sample will be collected 
into a $\mathrm{BD}{ }^{\circledR}$ EDTA (K2) (Becton, Dickinson and Company, Wokingham, Berkshire, UK) tube for plasma extraction.

Body Composition and Bone Mass Assessment: Total and regional body composition will be measured by DXA (Horizon W, Hologic Inc., Massachusetts, USA), with images analysed using preinstalled software for the calculation of lean and fat mass $(\mathrm{kg})$ as well as the percentage of fat mass. Due to the in-built analysis assumptions regarding the measurement of head composition, whole-body minus head data will be used. The coefficient of variation $(\% \mathrm{CV})$ for Hologic DXA fatfree soft tissue mass measurements ranges from $0.3-0.5 \%$, for fat mass, $2.5-5.9 \%$ for fat mass, and 2.5-5.7\% for lean mass, respectively[34]. Areal Bone Mineral Density (BMD) $\left(\mathrm{g} \cdot \mathrm{cm}^{-2}\right)$, Bone Mineral Content (BMC) $(\mathrm{g})$, and bone mineral area $\left(\mathrm{cm}^{2}\right)$ will be measured in the lumbar spine (L1-L4) and the left proximal femur by DXA and analysis conducted using the preinstalled machine software. Total lumbar spine, proximal femur, femoral neck and Ward's triangle will be analysed separately, with all scanning and analysis conducted by the same operator, with the $\% \mathrm{CV}$ reported as between 0.6-1.5 \% for Hologic DXA assessment of BMD[34].

Muscle Biopsy: Firstly, the vastus lateralis will be visualized via ultrasound (Siemens Acuson S3000 ${ }^{\mathrm{TM}}$, Sudbury, Suffolk, UK) to obtain an initial assessment of muscle depth for surgical safety. The area will be sterilized with a $3 \mathrm{ml}$ ChloraPrep ${ }^{\circledR}$ applicator twice (CareFusion, Becton, Dickinson and Company, Wokingham, Berkshire, UK) and the skin and overlying fascia will be infiltrated with a low-volume local anaesthetic (Lidocaine $50 \mathrm{mg} / 5 \mathrm{ml}$, Williams Medical Supplies, Rhymney, Caerphilly, UK). A small skin incision will be made using a sterile scalpel (Swann-Morton, Sheffield, South Yorkshire, UK). Using direct ultrasound visualisation four tissue samples will be collected with a single-use sterile 12-gauge biopsy needle (BARD Magnum ${ }^{\circledR}$ Disposable Core Biopsy Needles, $12 \mathrm{Gx} 10 \mathrm{~cm}$ or $12 \mathrm{Gx} 13 \mathrm{~cm}$, product code MN1210 or MN1213, Crawley, UK) via an 11-gauge coaxial needle (11Gx7 cm or $11 \mathrm{Gx} 10 \mathrm{~cm}$, product code $\mathrm{C} 1210 \mathrm{~B}$ or $\mathrm{C} 1213 \mathrm{~B})$ and placed in an alternative manner in either Qiagen ${ }^{\circledR}$ RNAlater RNA Stabilization Reagent (76106, Qiagen ${ }^{\circledR}$, Hilden, 
North Rhine-Westphalia, Germany) or Qiagen ${ }^{\circledR}$ Allprotect Tissue Reagent (76405) to preserve DNA, RNA and protein. This will complete the first part of the biopsy. The skin incision point will then be enlarged using a sterile scalpel (Swann-Morton, Sheffield, South Yorkshire, UK) and a sterile 6-gauge $(5.0 \mathrm{~mm})$ or 8-gauge $(4.0 \mathrm{~mm})$ University College Hospital (UCH) needle (Dixon's Surgical, Wickford, England) will be used to collect muscle samples. The UCH needle will be rotated and closed (with suction applied) four times to collect muscle samples under continuous ultrasound guidance, concluding the biopsy procedure. If the samples were insufficiently sized, further sampling will be performed.

Once the muscle is removed from the $\mathrm{UCH}$ needle it will be placed on a disposable freezing mould (Shandon ${ }^{\mathrm{TM}}$ Disposable Base Molds, Thermo Fisher Scientific) and its orientation analysed via a dissecting microscope for subsequent cutting in a cryostat. The muscle piece will be covered in Optimum Cutting Temperature Tissue Tek compound (AGR1180, Agar Scientific, Essex, UK) and immediately snap-frozen in isopentane and transferred to a $-80^{\circ} \mathrm{C}$ freezer for long-term storage. Muscle samples collected with the UCH needle will be used for immunohistochemical analysis (myonuclei and satellite cell number per fibre, fibre CSA and fibre type).

Handgrip Strength Test: Grip strength will be measured with a handgrip dynamometer (TAKEI 5401, TAKEI Scientific Instruments Co., Ltd, Japan) on both left and right hands 3 times each for a total of 6 repetitions with the mean result taken for each hand at each outcome point. The subject will be seated and their arm in a right-angled position.

Lower Body Anaerobic Power: Anaerobic power will be measured using the Wingate 30 seconds cycle test. The test measures lower body anaerobic power using a Monark bike ergometer (Monark Exercise AB, Vansbro, Sweden). The subject will perform a warm-up cycle of several minutes at 60 Revolutions Per Minute (RPM), the subject will then be asked to pedal as fast as possible for 30 seconds and verbally encouraged throughout. Within 3 seconds, a predetermined resistance of $75 \mathrm{~g} / \mathrm{kg}$ of the participant's body weight will be added to the ergometer. After the 30 seconds, the subject will 
be asked to cycle at 60 RPM for 3 minutes to warm down. Peak power (Watts [W]), relative peak power by body mass (W/kg), mean peak power (W), minimum peak power (W) and fatigue index will be determined from the decline in power.

$\dot{\mathbf{V}}_{\mathbf{o}_{2} \text { max }}: \dot{\mathrm{V}}_{\mathrm{O}_{2} \max }$ will be measured using a cycle ergometer (with increments of workload in Watts. The subject will wear a mask and the participants' $\dot{\mathrm{V}}_{2 \max }$ will be measured by oxygen and carbon dioxide analysers using breath by breath analysis (Quark CPET, COSMED Srl, Rome, Italy). Heart Rate will be measured every minute of the test using the Quark CPET electrocardiogram nodes. Additionally, the participant will point to a Rate of Perceived Exertion scale after each elevation of workload. The Subject will be considered to have reached their $\dot{\mathrm{V}}_{2 \max }$ if several of the following occurred: a plateau or 'peaking over' in oxygen uptake, maximal heart rate is reached, and volitional exhaustion.

Lung Function: The flow-volume loop spirometry test will be used to test the subject's lung function. Before the test, the equipment will be checked for calibration, the subject will then have been asked to not eat a large meal ( 2 hours), smoke ( $>1$ hour) and take part in vigorous exercise (30 mins) before arrival for this test. If the patient has an inhaler, they will be asked to take the inhaler before testing and this will be documented in tests results and analysis. The subject will then be asked to sit upright, feet flat on the floor uncrossed and tight clothing loosened. They will then be asked to inhale breath maximally, force their expiration as hard and as fast as possible before finally inhaling breath maximally again to complete the loop. The result will either classed as normal, obstructive, restrictive, fixed upper airway obstruction, variable extrathoracic obstruction or variable intrathoracic obstruction.

Physical Activity Assessment: The International Physical Activity Questionnaire and smart-watch technology will be used to estimate the time spent on various domains of physical activity. 


\subsection{Laboratory Visits}

Once recruited, participants will be required to report to the School of Pharmacy and Biomolecular

Sciences, the University of Brighton on 5 occasions: 2 prior ( 6 and 3 weeks), and 3 after $(3,6$, and 12 months) starting GAT. On each visit, participants will undergo an assessment of bone health, and body composition using DXA scanning, muscle physiology by biopsy, strength by handgrip dynamometer, lung function via spirometry, and sporting performance via $\dot{V}_{o_{2 m a x}}$ Testing on a cycle ergometer. All subjects will be asked to volunteer for a follow-up study some 18-24 months after starting GAT.

\subsection{Data Analysis}

Data will be presented as mean values with standard deviation unless otherwise stated. Changes in body composition, bone health, muscle physiology, strength, $\dot{V}_{o_{2 m a x}}$ and lung function will be analysed using a repeated measures ANCOVA using standard statistical packages (i.e., R), using baseline measures as a covariate to minimize the effect of differences in size on changes in the outcome measures.

\section{Abbreviations}

TTAS: Tavistock Transgender Athlete Study

GAT: Gender-affirming Treatment

DXA: Dual X-ray Absorptiometry

$\dot{\mathbf{V}} \mathbf{o}_{2 m a x}$ : Maximum Rate of Oxygen Consumption

RPM: Rotations Per Minute

BMD: Bone Mineral Density

W: Watts

Kg: Kilogram

G: grams

Cm: centimetres

UCH: University College Hospital

CSA: Cross Sectional Area

\section{Acknowledgements}


Blair Hamilton and Yannis Pitsiladis were responsible for the study conception. The first draft of the protocol was written by Blair Hamilton and all authors commented on subsequent versions of this protocol. All authors read and approved the final protocol.

6 Funding: This study is funded by the International Olympic Committee (IOC Grant 2018).

7 Ethical approval: Ethical approval has been obtained by the Health Research Authority (HRA) London - Brighton \& Sussex Research Ethics Committee and an amendment to this approval has been sought to include the additional measurements of DXA.

8 Declaration of Interest Statement: All authors declare that they have no conflict of interest or competing interests.

\section{References}

1. Jones BA, Arcelus J, Bouman WP, Haycraft E. Sport and transgender people: a systematic review of the literature relating to sport participation and competitive sport policies. Sports Medicine. 2017;47(4):701-16.

2. Kuyper L, Wijsen C. Gender identities and gender dysphoria in the Netherlands. Archives of sexual behavior. 2014;43(2):377-85.

3. NHS. Gender dysphoria. The National Health Service. 2020.

https://www.nhs.uk/conditions/gender-dysphoria/. Accessed 29th July 2020.

4. Coleman E, Bockting W, Botzer M, Cohen-Kettenis P, DeCuypere G, Feldman J et al. Standards of care for the health of transsexual, transgender, and gender-nonconforming people, version 7. International journal of transgenderism. 2012;13(4):165-232.

5. Wylie K, Barrett J, Besser M, Bouman WP, Bridgman M, Clayton A et al. Good practice guidelines for the assessment and treatment of adults with gender dysphoria. Sexual and Relationship Therapy. 2014;29(2):154-214. 
6. Hargie OD, Mitchell DH, Somerville IJ. 'People have a knack of making you feel excluded if they catch on to your difference': Transgender experiences of exclusion in sport. International Review for the Sociology of Sport. 2017;52(2):223-39.

7. Arcelus J, Bouman WP, Van Den Noortgate W, Claes L, Witcomb G, Fernandez-Aranda F. Systematic review and meta-analysis of prevalence studies in transsexualism. European Psychiatry. 2015;30(6):807-15.

8. Aitken M, Steensma TD, Blanchard R, VanderLaan DP, Wood H, Fuentes A et al. Evidence for an altered sex ratio in clinic-referred adolescents with gender dysphoria. The Journal of Sexual Medicine. 2015;12(3):756-63.

9. De Vries A, Kreukels B, T'sjoen G, Ålgars M, Mattila A. Increase of referrals to gender identity clinics: a European trend. Transgender healthcare in Europe Book of abstracts. 2015:10. 10. Sport CoAf. CAS 2014/A/3759 Dutee Chand v. Athletics Federation of India (AFI) \& The International Association of Athletics Federations (IAAF). 2014. https://www.doping.nl/media/kb/3317/CAS\%202014_A_3759\%20Dutee\%20Chand\%20vs.\%20AFI\% 20\%26\%20IAAF\%20\%28S\%29.pdf. Accessed 26th of February 2020.

11. Ziegler EM, Huntley TI. It Got too Tough to Not Be Me: Accommodating Transgender Athletes in Sport. JC \& UL. 2013;39:467.

12. Bianchi A. Transgender women in sport. Journal of the Philosophy of Sport. 2017;44(2):229-42. 13. Simon R. Deserving to be lucky: Reflections on the role of luck and desert in sports. Journal of the Philosophy of Sport. 2007;34(1):13-25.

14. Schultz J. Caster Semenya and the "question of too": Sex testing in elite women's sport and the issue of advantage. Quest. 2011;63(2):228-43.

15. Minsberg T. 'Boys Are Boys and Girls Are Girls': Idaho Is First State to Bar Some Transgender Athletes. The New York Times, Online. 2020. https://www.nytimes.com/2020/04/01/sports/transgender-idaho-ban-sports.html. Accessed April 17th 2020.

16. Heggie V. Subjective sex: science, medicine and sex tests in sports. Routledge handbook of sport, gender and sexuality. Routledge; 2014. p. 359-67. 
17. Bhasin S, Brito JP, Cunningham GR, Hayes FJ, Hodis HN, Matsumoto AM et al. Testosterone therapy in men with hypogonadism: an Endocrine Society clinical practice guideline. The Journal of Clinical Endocrinology \& Metabolism. 2018;103(5):1715-44.

18. Mauras N, Hayes V, Welch S, Rini A, Helgeson K, Dokler M et al. Testosterone deficiency in young men: marked alterations in whole body protein kinetics, strength, and adiposity. The Journal of Clinical Endocrinology \& Metabolism. 1998;83(6):1886-92.

19. Elbers J, Asscheman H, Seidell J, Gooren LJ. Effects of sex steroid hormones on regional fat depots as assessed by magnetic resonance imaging in transsexuals. American Journal of PhysiologyEndocrinology and Metabolism. 1999;276(2):E317-E25.

20. Gooren LJ, Bunck MC. Transsexuals and competitive sports. European Journal of Endocrinology. 2004;151(4):425-30.

21. T'Sjoen G, Weyers S, Taes Y, Lapauw B, Toye K, Goemaere S et al. Prevalence of low bone mass in relation to estrogen treatment and body composition in male-to-female transsexual persons. Journal of Clinical Densitometry. 2009;12(3):306-13.

22. Wiik A, Lundberg T, Rullman E, Andersson D, Holmberg M, Mandić M et al. Muscle Strength, Size, and Composition Following 12 Months of Gender-affirming Treatment in Transgender Individuals. The Journal of clinical endocrinology and metabolism. 2020;105(3).

23. Storer TW, Magliano L, Woodhouse L, Lee ML, Dzekov C, Dzekov J et al. Testosterone dosedependently increases maximal voluntary strength and leg power, but does not affect fatigability or specific tension. The Journal of Clinical Endocrinology \& Metabolism. 2003;88(4):1478-85.

24. Jacobeit J, Gooren L, Schulte H. Safety aspects of 36 months of administration of long-acting intramuscular testosterone undecanoate for treatment of female-to-male transgender individuals. European journal of endocrinology. 2009;161(5):795.

25. Lilja M, Mandić M, Apró W, Melin M, Olsson K, Rosenborg S et al. High doses of antiinflammatory drugs compromise muscle strength and hypertrophic adaptations to resistance training in young adults. Acta Physiologica. 2018;222(2):e12948. 
26. Roberts TA, Smalley J, Ahrendt D. Effect of gender affirming hormones on athletic performance in transwomen and transmen: implications for sporting organisations and legislators. British Journal of Sports Medicine. 2020:bjsports-2020-102329. doi:10.1136/bjsports-2020-102329.

27. Unnanuntana A, Gladnick BP, Donnelly E, Lane JM. The assessment of fracture risk. The Journal of Bone and Joint Surgery American volume. 2010;92(3):743.

28. Bruusgaard JC, Johansen I, Egner I, Rana Z, Gundersen K. Myonuclei acquired by overload exercise precede hypertrophy and are not lost on detraining. Proceedings of the National Academy of Sciences. 2010;107(34):15111-6.

29. Bruusgaard JC, Gundersen K. In vivo time-lapse microscopy reveals no loss of murine myonuclei during weeks of muscle atrophy. The Journal of clinical investigation. 2008;118(4):1450-7.

30. Snijders T, Aussieker T, Holwerda A, Parise G, van Loon LJ, Verdijk LB. The concept of skeletal muscle memory: Evidence from animal and human studies. Acta Physiologica. 2020:e13465.

31. Gundersen K. Muscle memory and a new cellular model for muscle atrophy and hypertrophy. Journal of Experimental Biology. 2016;219(2):235-42.

32. Seaborne RA, Strauss J, Cocks M, Shepherd S, O’Brien TD, Van Someren KA et al. Human skeletal muscle possesses an epigenetic memory of hypertrophy. Scientific reports. 2018;8(1):1-17. 33. Murach KA, Mobley CB, Zdunek CJ, Frick KK, Jones SR, McCarthy JJ et al. Muscle memory: myonuclear accretion, maintenance, morphology, and miRNA levels with training and detraining in adult mice. Journal of cachexia, sarcopenia and muscle. 2020.

34. Bilsborough JC, Greenway K, Opar D, Livingstone S, Cordy J, Coutts AJ. The accuracy and precision of DXA for assessing body composition in team sport athletes. Journal of sports sciences. 2014;32(19):1821-8. 